\title{
Rapid Detection of Polymyxin-Resistant Enterobacteriaceae from Blood Cultures
}

\author{
Aurélie Jayol, ${ }^{a, b, c}$ Véronique Dubois, ${ }^{c}$ Laurent Poirel, ${ }^{a, b}$ Patrice Nordmann ${ }^{a, b, d}$ \\ Emerging Antibiotic Resistance Unit, Medical and Molecular Microbiology, Department of Medicine, University of Fribourg, Fribourg, Switzerlanda; INSERM European Unit \\ (LEA Paris, France), University of Fribourg, Fribourg, Switzerland ${ }^{\text {b; }}$ CNRS UMR5234, University of Bordeaux, Bordeaux, France ${ }^{\text {; }}$, University of Lausanne and University \\ Hospital Center, Lausanne, Switzerland ${ }^{\mathrm{d}}$
}

Enterobacterial strains resistant to polymyxins are being increasingly reported worldwide. The conventional methods for detection of colistin-resistant isolates such as broth microdilution remain time-consuming ( 24 to $48 \mathrm{~h}$ ), and methods such as disc diffusion and Etest are not reliable. Recently, the rapid polymyxin NP test was developed for rapid identification of polymyxin-resistant Enterobacteriaceae. This test is based on the detection of glucose metabolism related to bacterial growth in the presence of a defined concentration of colistin (or polymyxin B). The formation of acid metabolites is evidenced by a color change of a pH indicator (red phenol) in less than $2 \mathrm{~h}$. In this study, the polymyxin NP test was evaluated for detection of colistin-resistant $E n$ terobacteriaceae directly from blood cultures. The test was performed with 73 blood culture sets (either spiked or clinical blood cultures) with various enterobacterial species. The test exhibited excellent discrimination between polymyxin-resistant and polymyxin-susceptible enterobacterial isolates, and results are obtained from blood cultures within $4 \mathrm{~h}$. It is easy to perform and requires neither subculture nor a centrifugation step. This test is rapid, specific, and sensitive and allows early identification of polymyxin-resistant Enterobacteriaceae directly from blood cultures.

T he spread of carbapenemase-producing Enterobacteriaceae has now been reported worldwide (1). Considering the paucity of antibiotics that are effective against those multidrug-resistant bacteria, it is of utmost importance to preserve the clinical efficacy of last-resort antibiotics such as polymyxins (colistin and polymyxin B) (2). However, polymyxin-resistant Enterobacteriaceae are being increasingly reported worldwide, exhibiting either chromosomal mutations (3) or plasmid-mediated $m c r-1$ gene acquisition (4). The methods available to determine polymyxin susceptibility require isolation and growth of the bacteria from the infected samples, and the susceptibility testing methods add, at least, an additional delay of $18 \mathrm{~h}$ to obtain the results (5). Moreover, routine methods used in the clinical microbiology laboratory (disk diffusion, Etest strips, and some automated systems such as the Vitek2 and the MicroScan) are not reliable for detecting polymyxin-resistant isolates (5-9). Molecular tests are not effective for determining polymyxin resistance due to the variety of genes that may be altered or truncated as a source of chromosomal resistance (3).

Rapid identification and antimicrobial susceptibility testing are essential for guiding clinicians in the selection of the most appropriate treatment for patients with bloodstream infections. The mortality rates from sepsis in intensive care units range from 20 to $60 \%$ worldwide. Inappropriate initial antimicrobial therapy for septic shock occurs in about $20 \%$ of patients, is associated with a 5-fold reduction in survival (10), and is responsible for an increased length of hospital stay (11). This is largely due to the fact that its takes several days to obtain the susceptibility pattern of the bacteria responsible for the septicemia. In Italy, a large hospital outbreak of colistin-resistant and carbapenemase (KPC)-producing Klebsiella pneumoniae isolates, correlating with the local increased colistin consumption, has been recently described (12). Therefore, there is a need for a rapid detection of polymyxinresistant isolates from blood cultures.

Recently, a diagnostic test (the rapid polymyxin NP test) based on the detection of glucose metabolism related to bacterial growth in the presence of colistin has been developed to rapidly identify $(<2 \mathrm{~h})$ polymyxin-resistant enterobacterial strains from colonies grown on solid media (13). This test yields sensitivity and specificity values of $99.3 \%$ and $95.4 \%$, respectively. It has not yet been evaluated with clinical samples.

The aim of this study was to determine the ability of the rapid polymyxin NP test to detect polymyxin-resistant Enterobacteriaceae from blood cultures. First, we evaluated this test with blood cultures spiked with colistin-resistant enterobacterial strains exhibiting various mechanisms of resistance (intrinsic or acquired, chromosomal, and plasmid-mediated). Then, the test was performed prospectively on clinical blood cultures positive for Gramnegative bacteria.

\section{MATERIALS AND METHODS}

Spiked blood cultures. The panel of strains used for spiking blood cultures included a single reference strain susceptible to colistin (Escherichia coli ATCC 25922) and 52 colistin-resistant enterobacterial strains (MICs of colistin ranging from 4 to $>128 \mu \mathrm{g} / \mathrm{ml}$ ). Among the latter strains, 5 strains exhibited an intrinsic polymyxin resistance trait (Proteus spp., $n=$ 2; Providencia spp., $n=1$; Morganella spp., $n=1$; and Serratia spp., $n=$ 1 ), and 47 strains presented an acquired resistance trait and belonged to various species (E. coli, $n=11$; Klebsiella spp., $n=31$; Enterobacter spp., $n=4$; and Hafnia alvei, $n=1$ ). Some of the strains had previously been

Received 27 April 2016 Returned for modification 18 May 2016

Accepted 8 June 2016

Accepted manuscript posted online 15 June 2016

Citation Jayol A, Dubois V, Poirel L, Nordmann P. 2016. Rapid detection of polymyxin-resistant Enterobacteriaceae from blood cultures. J Clin Microbiol 54:2273-2277. doi:10.1128/JCM.00918-16.

Editor: P. Bourbeau, St. James

Address correspondence to Patrice Nordmann, patrice.nordmann@unifr.ch. Copyright $\odot$ 2016, American Society for Microbiology. All Rights Reserved. 
TABLE 1 Rapid polymyxin NP test results for spiked blood cultures and clinical blood cultures

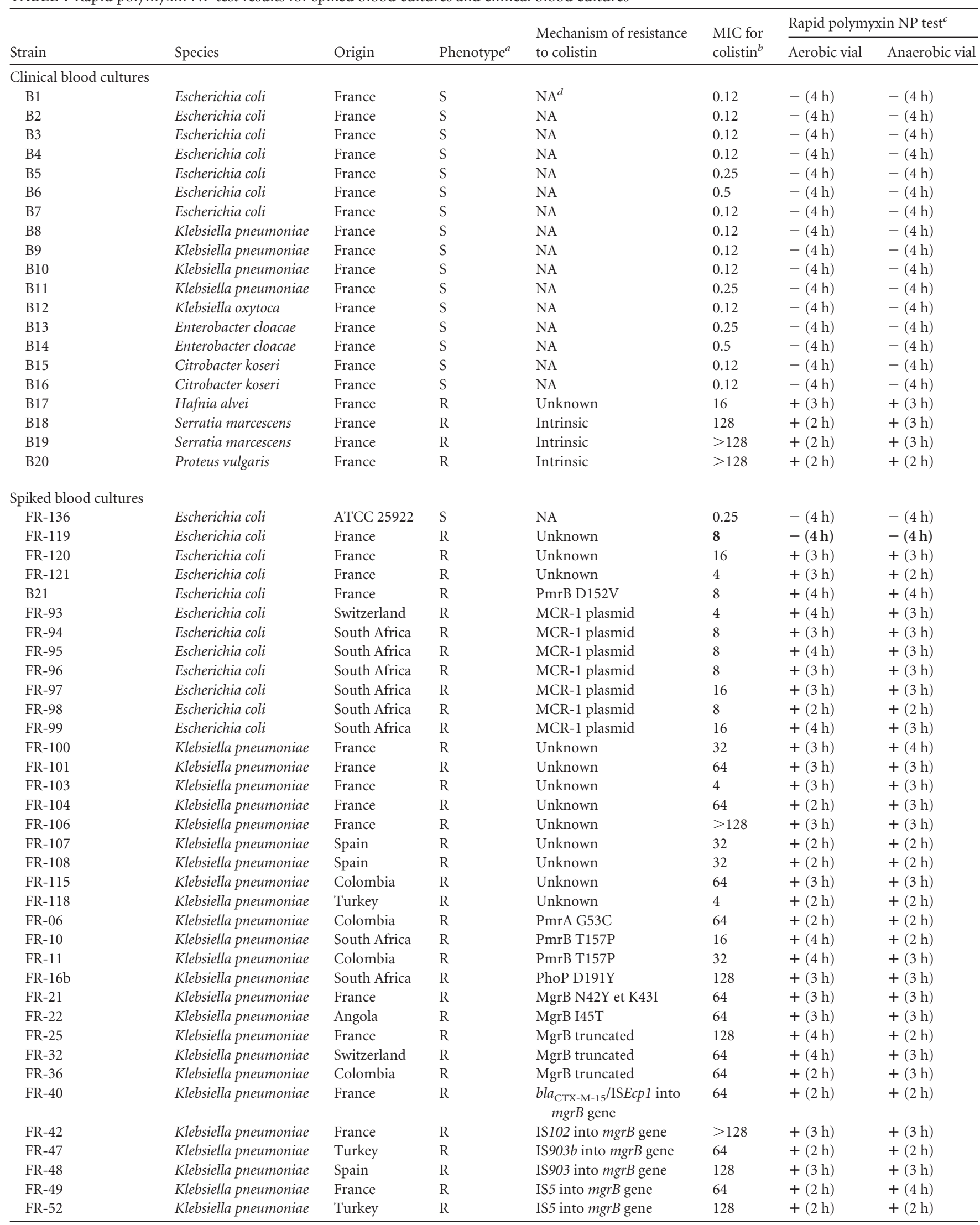


TABLE 1 (Continued)

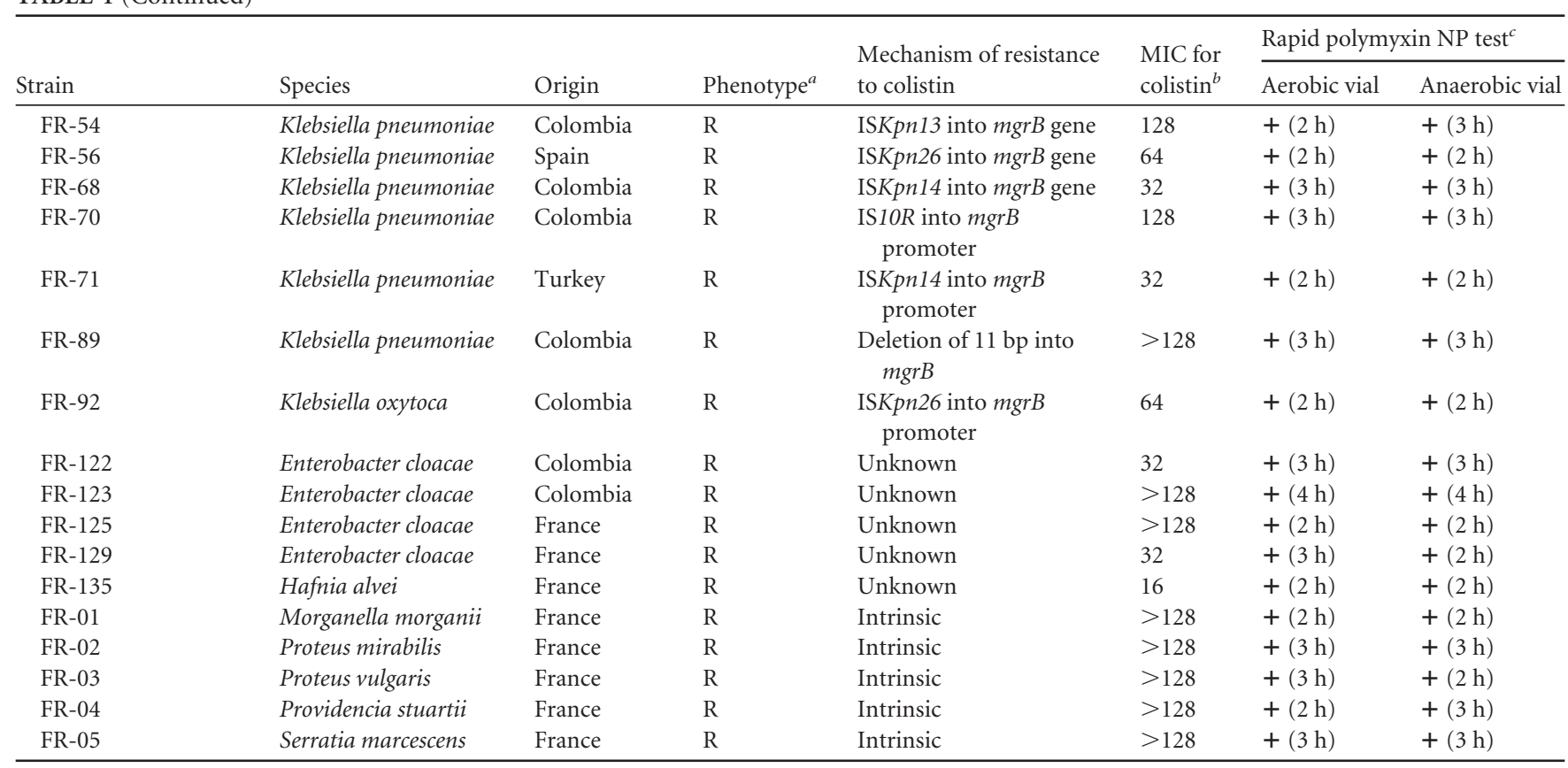

${ }^{a} \mathrm{~S}$, susceptible; $\mathrm{R}$, resistant.

${ }^{b}$ MIC values of colistin: the breakpoint value for susceptible is $\leq 2 \mu \mathrm{g} / \mathrm{ml}$ and for resistance is $>2 \mu \mathrm{g} / \mathrm{ml}$ for Enterobacteriaceae according to the EUCAST (23).

${ }^{c}$ Discrepant results obtained with the rapid polymyxin NP test compared to the broth dilution method are in bold. - , negative; + , positive.

${ }^{d} \mathrm{NA}$, not applicable.

characterized for their polymyxin resistance mechanisms at the molecular level (13-19).

To spike the blood cultures, the colistin-resistant enterobacterial strains were subcultured onto Luria-Bertani agar overnight. Then, aerobic and anaerobic blood culture vials without charcoal (BacT/Alert FA Plus and BacT/Alert FN Plus, respectively; bioMérieux, Marcy l'Etoile, France) containing $10 \mathrm{ml}$ of sterile human blood were inoculated with $10^{3} \mathrm{CFU} / \mathrm{ml}$ of the bacterial strain to be tested. This inoculum was prepared by diluting a $0.5 \mathrm{McF}$ arland standard suspension (about $10^{8} \mathrm{CFU} / \mathrm{ml}$ ) in $0.9 \% \mathrm{NaCl}$. Then, blood culture vials were incubated until a positive blood culture was detected by the BacT/Alert 3D blood culture system. With this protocol, the time for detecting the positivity of the blood cultures ranged from 6 to $15 \mathrm{~h}$, and the final inoculum ranged from $10^{7}$ to $10^{9} \mathrm{CFU} / \mathrm{ml}$, as previously reported (20).

Clinical blood cultures. All sets of positive blood cultures recovered during the November 2015 to February 2016 period of time from nonduplicate patients that were detected as positive by the BacT/Alert 3D blood culture system (bioMérieux) and stained as Gram-negative were included. Both aerobic and anaerobic vials were tested. Species identification was carried out with the matrix-assisted laser desorption ionizationtime of flight mass spectrometry (MALDI-TOF MS) Biotyper system (Bruker Corporation, Bremen, Germany) after $3 \mathrm{~h}$ of incubation of the blood culture on chocolate agar. Positive blood cultures with nonenterobacterial isolates or with a mix of bacteria were excluded from the testing. Overall, a total of 20 monomicrobial blood cultures positive for enterobacterial isolates were tested (Table 1).

Colistin susceptibility testing. Colistin susceptibility testing was performed by determination of MICs using the broth microdilution method (BMD) according to the CLSI guidelines $(21,22)$. The BMD method was carried out with colonies grown on chocolate agar after incubation of the positive blood cultures. The EUCAST guidelines (version 2015) were used for categorical agreement (23).

Detection of colistin resistance from positive blood cultures using the rapid polymyxin NP test. For each positive blood culture tested (spiked or clinical blood culture), $50 \mu \mathrm{l}$ of blood culture was mixed with
$450 \mu \mathrm{l}$ of sterile $0.9 \% \mathrm{NaCl}$ (10-fold dilution). The rapid polymyxin NP test was then performed as described with bacterial colonies (13). Briefly, $50 \mu \mathrm{l}$ of the 10 -fold dilution of the blood culture was mixed with (i) $150 \mu \mathrm{l}$ of colistin-free polymyxin NP solution and (ii) $150 \mu$ l of polymyxin NP solution containing $5 \mu \mathrm{g} / \mathrm{ml}$ of colistin sulfate (Sigma-Aldrich, Buchs, Switzerland) in a 96-well polystyrene plate (reference no. 82.1582.001; Sarstedt, Nümbrecht, Germany) (Fig. 1). The final concentration of colistin was $3.75 \mu \mathrm{g} / \mathrm{ml}$ in each well. In parallel, $50 \mu \mathrm{l}$ of $0.9 \% \mathrm{NaCl}$ was mixed with the polymyxin NP solution to verify the absence of medium contamination. The plate was incubated at $35 \pm 2{ }^{\circ} \mathrm{C}$ in ambient air for $4 \mathrm{~h}$, and visual inspection was performed each hour to observe the color change. Test results were interpreted by technicians who were blinded to the sample identity. The test was considered negative when the well containing the mix with colistin remained orange and positive when it turned from orange to yellow (Fig. 1). The test was considered noninterpretable when the colistin-free well remained orange (absence of glucose metabolization) (Fig. 1). E. coli ATCC 25922 and a Morganella morganii strain were used as negative and positive controls, respectively.

\section{RESULTS AND DISCUSSION}

To determine the performance of the rapid polymyxin NP test directly from blood cultures, 73 blood culture sets (aerobic and anaerobic vials) positive for enterobacterial strains were tested, including monomicrobial clinical samples and blood cultures spiked with colistin-resistant isolates presenting various mechanisms of resistance (intrinsic, acquired chromosomally encoded, plasmid-mediated, and unknown mechanisms). The results for the rapid polymyxin NP test were compared with the MICs determined by the BMD method taken as the gold standard.

The 52 blood cultures spiked with enterobacterial strains resistant to colistin gave positive results in 2 to $4 \mathrm{~h}$ (Table 1 ), except for a single colistin-resistant $E$. coli isolate that also gave a negative result by using the rapid polymyxin NP test performed from col- 


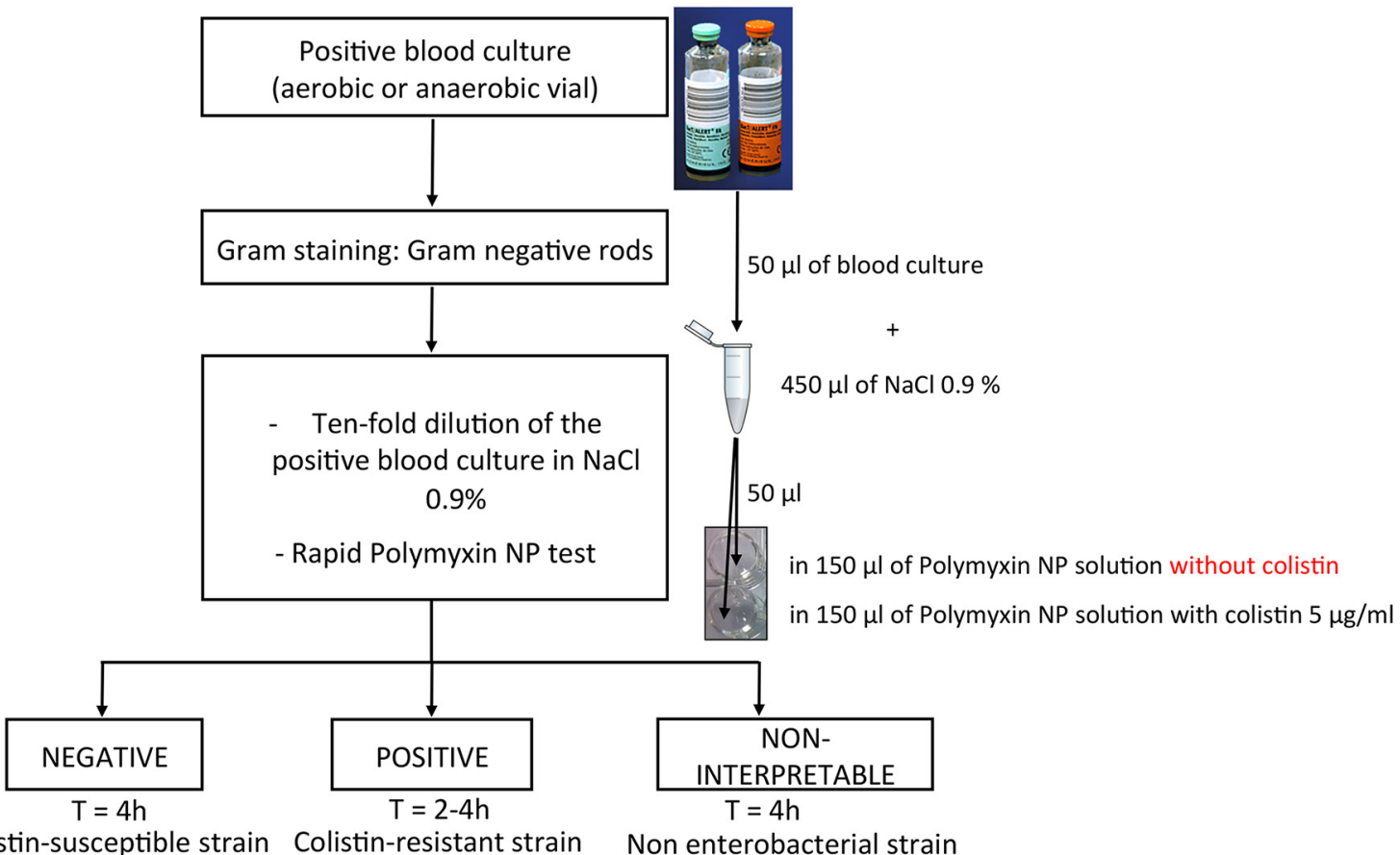

Colistin-susceptible strain Colistin-resistant strain Non enterobacterial strain

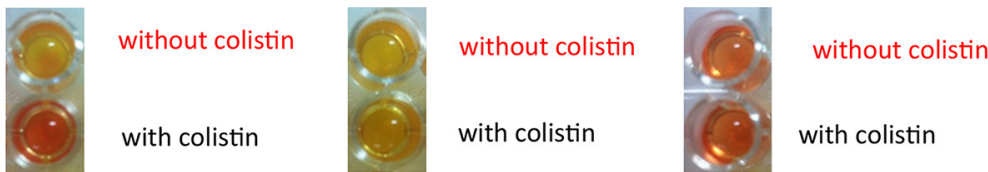

FIG 1 Strategy for identification of colistin-resistant Enterobacteriaceae from blood cultures using the rapid polymyxin NP test. T, time.

onies (13). The mechanism of colistin resistance of this single isolate remains unknown.

Twenty positive clinical blood culture sets detected by the BacT/Alert 3D blood culture system were also tested. Among them, four were positive with colistin-resistant enterobacterial isolates in 2 to $4 \mathrm{~h}$ of incubation: three isolates belonged to species that are naturally resistant to colistin (two Serratia marcescens isolates and a single Proteus vulgaris isolate) and a single $H$. alvei isolate showed acquired resistance to colistin. The rapid polymyxin NP test gave positive results for all of those isolates (Table 1). Sixteen blood cultures were grown with enterobacterial isolates belonging to genera naturally susceptible to colistin (E. coli, Klebsiella, Enterobacter, and Citrobacter). None of them presented acquired resistance to colistin (the MICs of colistin ranging from 0.125 to $0.5 \mu \mathrm{g} / \mathrm{ml}$ ) and the rapid polymyxin NP test gave negative results. Positive blood cultures with nonfermenting bacterial isolates (Acinetobacter spp. and Pseudomonas spp.) gave noninterpretable results (Fig. 1).

A series of other conditions (variable blood culture dilutions or conditions of atmosphere incubation) were tested but gave less optimal results. Indeed, the test performed without blood culture dilution revealed that the excess of blood prevented the optimal reading of color changes. We did not observe spontaneous lysis of the blood cultures that might possibly have interfered with the results of the test. Other blood culture dilutions $(1 / 5$ or $1 / 100)$ gave less rapid positive results (data not shown). Incubation under $\mathrm{CO}_{2}$ gave false-positive results because of excessive acidification of the polymyxin NP solution, as opposed to results obtained with the rapid polymyxin NP test performed with bacterial colonies (13).

This test performed from blood cultures may have the same limitation as the rapid polymyxin NP test performed from bacterial colonies (13), i.e., the lack of detection of heteroresistant isolates with low MICs to polymyxins by BMD. This limitation has to be further explored.

In conclusion, the rapid polymyxin NP test from positive blood cultures showed good performances for the detection of enterobacterial isolates resistant to colistin. It shortens the delay in obtaining results to ca. $4 \mathrm{~h}$ versus $48 \mathrm{~h}$ for standard antibiotic susceptibility testing ( $24 \mathrm{~h}$ for obtaining a bacterial culture and 24 additional hours for susceptibility testing). This test may be implemented for routine testing of blood cultures and is best when combined with a rapid identification technique such as MALDITOF technology. It is cost-effective since it avoids in particular the time-consuming steps of manual determination of MIC values (e.g., powder weighing and dilution). As highlighted above, this test allows rapid identification of polymyxin-resistant Enterobacteriaceae responsible for bacteremia, which is not possible with molecular techniques, considering the complexity and the diversity of the polymyxin resistance mechanisms.

Early detection of colistin-resistant strains might contribute to early initiation of appropriate antibiotic therapy for septic patients infected with multidrug-resistant Enterobacteriaceae. In countries where carbapenemase-producing enterobacterial isolates are endemic (e.g., Greece and Italy), implementation of the test might contribute to early initiation of appropriate antibiotic 
therapy. Its use may preserve polymyxin as the last-resort antibiotherapy. In addition, implementation of contact isolation precautions for carriers of polymyxin-resistant isolates could prevent the development of outbreaks with those multidrug-resistant isolates.

\section{ACKNOWLEDGMENTS}

This work was financed by the University of Fribourg, Switzerland.

An international patent form has been filed on behalf of the University of Fribourg (Switzerland) corresponding to the rapid polymyxin NP test.

\section{FUNDING INFORMATION}

This work, including the efforts of Aurelie Jayol, Véronique Dubois, Laurent Poirel, and Patrice Nordmann, was funded by University of Fribourg, Switzerland.

\section{REFERENCES}

1. Nordmann P, Poirel L. 2014. The difficult-to-control spread of carbapenemase producers among Enterobacteriaceae worldwide. Clin Microbiol Infect 20:821-830. http://dx.doi.org/10.1111/1469-0691.12719.

2. Livermore DM, Warner M, Mushtaq S, Doumith M, Zhang J, Woodford N. 2011. What remains against carbapenem-resistant Enterobacteriaceae? Evaluation of chloramphenicol, ciprofloxacin, colistin, fosfomycin, minocycline, nitrofurantoin, temocillin and tigecycline. Int J Antimicrob Agents 37:415-419. http://dx.doi.org/10.1016/j.ijantimicag.2011.01.012.

3. Olaitan AO, Morand S, Rolain JM. 2014. Mechanisms of polymyxin resistance: acquired and intrinsic resistance in bacteria. Front Microbiol 5:643. http://dx.doi.org/10.3389/fmicb.2014.00643.

4. Liu YY, Wang Y, Walsh TR, Yi LX, Zhang R, Spencer J, Doi Y, Tian G, Dong B, Huang X, Yu LF, Gu D, Ren H, Chen X, Lv L, He D, Zhou H, Liang Z, Liu JH, Shen J. 2016. Emergence of plasmid-mediated colistin resistance mechanism MCR-1 in animals and human beings in China: a microbiological and molecular biological study. Lancet Infect Dis 16:161168. http://dx.doi.org/10.1016/S1473-3099(15)00424-7.

5. Humphries RM. 2015. Susceptibility testing of the polymyxins: where are we now? Pharmacotherapy 35:22-27. http://dx.doi.org/10.1002/phar .1505 .

6. Lo-Ten-Foe JR, de Smet AM, Diederen BM, Kluytmans JA, van Keulen PH. 2007. Comparative evaluation of the Vitek 2, disk diffusion, Etest, broth microdilution, and agar dilution susceptibility testing methods for colistin in clinical isolates, including heteroresistant Enterobacter cloacae and Acinetobacter baumannii strains. Antimicrob Agents Chemother 51: 3726-3730. http://dx.doi.org/10.1128/AAC.01406-06.

7. Maalej SM, Meziou MR, Rhimi FM, Hammami A. 2011. Comparison of disc diffusion, Etest and agar dilution for susceptibility testing of colistin against Enterobacteriaceae. Lett Appl Microbiol 53:546-551. http://dx.doi .org/10.1111/j.1472-765X.2011.03145.x.

8. Tan TY, Ng SY. 2007. Comparison of Etest, Vitek and agar dilution for susceptibility testing of colistin. Clin Microbiol Infect 13:541-544. http: //dx.doi.org/10.1111/j.1469-0691.2007.01708.x.

9. Perez LR. 2015. Evaluation of polymyxin susceptibility profile among KPC-producing Klebsiella pneumoniae using Etest and MicroScan WalkAway automated system. APMIS 123:951-954. http://dx.doi.org/10.1111 /apm.12438.

10. Kumar A, Ellis P, Arabi Y, Roberts D, Light B, Parrillo JE, Dodek P, Wood G, Kumar A, Simon D, Peters C, Ahsan M, Chateau D, Cooperative Antimicrobial Therapy of Septic Shock Database Research
Group. 2009. Initiation of inappropriate antimicrobial therapy results in a fivefold reduction of survival in human septic shock. Chest 136:12371248. http://dx.doi.org/10.1378/chest.09-0087.

11. Garnacho-Montero J, Ortiz-Leyba C, Herrera-Melero I, Aldabó-Pallás T, Cayuela-Dominguez A, Marquez-Vacaro JA, Carbajal-Guerrero J, Garcia-Garmendia JL. 2008. Mortality and morbidity attributable to inadequate empirical antimicrobial therapy in patients admitted to the ICU with sepsis: a matched cohort study. J Antimicrob Chemother 61:436441. http://dx.doi.org/10.1093/jac/dkm460.

12. Giani T, Arena F, Vaggelli G, Conte V, Chiarelli A, Henrici De Angelis L, Fornaini R, Grazzini M, Niccolini F, Pecile P, Rossolini GM. 2015. Large nosocomial outbreak of colistin-resistant, carbapenemaseproducing Klebsiella pneumoniae traced to clonal expansion of an $m g r B$ deletion mutant. J Clin Microbiol 53:3341-2234. http://dx.doi.org/10 .1128/JCM.01017-15.

13. Nordmann P, Jayol A, Poirel L. 2016. Rapid detection of polymyxin resistance in Enterobacteriaceae. Emerg Infect Dis 22:1038-1043. http://dx .doi.org/10.3201/eid2206.151840.

14. Jayol A, Nordmann P, Brink A, Poirel L. 2015. Heteroresistance to colistin in Klebsiella pneumoniae associated with alterations in the PhoPQ regulatory system. Antimicrob Agents Chemother 59:2780-2784. http: //dx.doi.org/10.1128/AAC.05055-14.

15. Jayol A, Nordmann P, Desroches M, Decousser JW, Poirel L. 2016. Acquisition of broad-spectrum cephalosporin resistance leading to colistin resistance in Klebsiella pneumoniae. Antimicrob Agents Chemother 60:3199-3201. http://dx.doi.org/10.1128/AAC.00237-16.

16. Jayol A, Poirel L, Brink A, Villegas MV, Yilmaz M, Nordmann P. 2014. Resistance to colistin associated with a single amino acid change in protein PmrB among Klebsiella pneumoniae isolates of worldwide origin. Antimicrob Agents Chemother 58:4762-4766. http://dx.doi.org/10.1128/AAC .00084-14.

17. Jayol A, Poirel L, Villegas MV, Nordmann P. 2015. Modulation of $m g r B$ gene expression as a source of colistin resistance in Klebsiella oxytoca. Int J Antimicrob Agents 46:108-110. http://dx.doi.org/10.1016/j.ijantimicag .2015.02.015

18. Poirel L, Jayol A, Bontron S, Villegas MV, Ozdamar M, Türkoglu S, Nordmann P. 2015. The $m g r B$ gene as a key target for acquired resistance to colistin in Klebsiella pneumoniae. J Antimicrob Chemother 70:75-80. http://dx.doi.org/10.1093/jac/dku323.

19. Poirel L, Kieffer N, Liassine N, Thanh D, Nordmann P. 2016. Plasmidmediated carbapenem and colistin resistance in a clinical isolate of Escherichia coli. Lancet Infect Dis 16:281-282. http://dx.doi.org/10.1016/S1473 -3099(16)00007-4.

20. Dortet L, Bréchard L, Poirel L, Nordmann P. 2014. Rapid detection of carbapenemase-producing Enterobacteriaceae from blood cultures. Clin Microbiol Infect 20:340-344. http://dx.doi.org/10.1111/1469 -0691.12318.

21. Clinical and Laboratory Standards Institute. 2012. Methods for dilution of antimicrobial susceptibility tests for bacteria that grow aerobically; approved standard-9th ed. CLSI document M07-A9. Clinical and Laboratory Standards Institute, Wayne, PA.

22. Clinical and Laboratory Standards Institute. 2015. Performance standards for antimicrobial susceptibility testing. CLSI document M100-S25. Clinical and Laboratory Standards Institute, Wayne, PA.

23. European Committee on Antimicrobial Susceptibility Testing. 2014. Breakpoint tables for interpretation of MICs and zone diameters, version 2.0. European Committee on Antimicrobial Susceptibility Testing, Basel, Switzerland. 